\title{
C. COBENGE \\ GRUPOS DE TRABALHO NO PROJETO DE EXTENSÃO E A PERCEPÇÃO DOS ALUNOS PARA MELHORAR A COORDENAÇÃO DE TAREFAS
}

DOI: $10.37702 / 2175-957 X . C O B E N G E .2021 .3478$

Francisco de Salles Cintra Gomes - salles@puc-campinas.edu.br

Pontifícia Universidade Católica de Campinas

Rod. Dom Pedro I km 136 sem número

13086-900 - CAMPINAS - SP

Lucas Grass Beraldo - grassberaldo@gmail.com

Pontifícia Universidade Católica de Campinas

Avenida Brunoro de gasperi 256

13044-165 - Campinas - SP

Ludmila Cordeiro Lopes Vitoriano - ludlops20@gmail.com

Pontifícia Universidade Católica de Campinas

Rua Antônio Ferragut 110

13289-328 - Vinhedo - SP

Victor Caodaglio do Amaral Gurgel - victorgurgel@outlook.com.br Pontifícia Universidade Católica de Campinas

Rua Comendador Gumercindo Barranqueiros 640

13211-410 - Jundiaí - SP

Victor Luiz Ildebrand de Souza - victor_ildebrand@hotmail.com

Pontifícia Universidade Católica de Campinas

Rua Manoel Ferreira de Souza 52

13056-151 - Campinas - SP

Marcela Pereira Alferes - marcelaalferes11@gmail.com

Pontifícia Universidade Católica de Campinas PUC Campinas

Rua Jaracatia 165

05754-070 - São Paulo - SP

Resumo: O Projeto de Extensão voltado à Eficiência Energética teve em 2020 um 
aumento expressivo no número de alunos participantes, que passou de 8 alunos, em 2019, para 35 alunos em 2020. A maneira de dedicação oferecida foi dividir as ações em grupos de trabalho. Com a curricularização da extensão universitária o número de participantes deverá aumentar significativamente. $O$ objetivo deste artigo é apresentar as percepções dos alunos que participaram dos grupos de trabalho do Projeto de Extensão. Como metodologia foi elaborado um questionário para que os alunos expusessem seus pontos de vista. Nos resultados são apresentados os dados dos questionários com a experiência dos alunos sobre $o$ trabalho realizado em equipe, e suas sugestões para melhorar a coordenação de produtividade. Como conclusão, a maioria dos alunos prefere trabalhar em equipes menores. A formação de grupos de trabalho dá condições para a prática da liderança de equipes e o favorece a capacidade para trabalhar em equipes multidisciplinares, bem como, expor ideias para a execução do trabalho, cooperando com o desenvolvimento colaborativo. Faz parte integrante das ações da Extensão a produção conjunta de material de natureza técnico-cultural, para melhorar a autonomia do público-alvo e facilitar maior abrangência do conhecimento adquirido a outros públicos. Os Trabalhos de Extensão são realizados em conjunto com a Pró-Reitoria de Extensão e Assuntos Comunitários da Pontifícia Universidade Católica de Campinas, com a participação das comunidades parceiras.

Palavras-chave: Extensão Universitária. Formação Acadêmica do Aluno. Eficiência Energética. Competências na Engenharia. Grupos de Trabalho. 


\section{INTRODUÇÃO}

Este artigo conta com a participação de alunos voluntários que atuaram no Projeto de Extensão voltado à Eficiência Energética em 2020 e vivenciaram a divisão das tarefas e das ações da extensão. O número de voluntários participantes passou de 8, em 2019, para 35 em 2020. A maneira de trabalhar adotada foi dividir as ações em grupos menores que favorecessem o modo de trabalho colaborativo.

Para este artigo foi elaborado um questionário voltado aos alunos que participaram dos vários grupos de trabalho para que, através de suas percepções, fossem vistos caminhos ou possibilidades para melhorar a coordenação de tarefas.

Com a curricularização da extensão universitária o número de participantes deverá aumentar significativamente e novas experiências na coordenação serão fundamentais para colaborar com bons êxitos nas ações da extensão. As experiências adquiridas e a percepção dos alunos envolvidos poderão auxiliar na execução de outros trabalhos com muitos participantes.

O trabalho em grupo possibilita o exercício e a prática de várias competências, tais como: liderança, responsabilidade, planejamento, orientação, acompanhamento, execução, treinamento, dentre outras. Na Engenharia é comum a divisão de tarefas e a formação de equipes ou grupos de trabalho.

A prática da extensão com alunos dos cursos de Engenharia além de colocá-los no exercício de tarefas além da sala de aula, oferece também oportunidades de vivenciar diferentes realidades.

\section{PROJETO DE EXTENSÃO}

$\mathrm{Na}$ Pontifícia Universidade Católica de Campinas (PUC-Campinas), os docentes têm a possibilidade de propor um Trabalho de Extensão para o biênio ou para um ano com a participação de alunos. Um plano com descrição detalhada é elaborado, contendo os objetivos e as atividades a serem desenvolvidas.

Em 2020, houve o incentivo da universidade para que mais alunos voluntários pudessem participar nos trabalhos. A participação passou a ser em fluxo contínuo, podendo o aluno ingressar ou sair em qualquer momento, sem limite do número de participantes. A dedicação de horas semanais pode ser diferente para cada aluno, podendo ser no máximo 12 horas por semana.

\subsection{Projeto de extensão voltado à eficiência energética}

No biênio 2020-2021 está sendo realizado o projeto de extensão "A Inovação Social e o compromisso com a Sustentabilidade através de ações voltadas ao Uso Racional de Energia e Eficiência Energética", que tem como principal objetivo "desenvolver processos e/ou produtos, visando a sustentabilidade ambiental, econômica e social, inclusive a geração de renda de populações vulneráveis". Participam desse trabalho um docente extensionista e alunos voluntários dos cursos de engenharia.

O público-alvo conta com a participação de pessoas em situação de vulnerabilidade social vinculado às Comunidades das Paróquias Jesus Cristo Libertador da região do Campo Grande (Campinas-SP).

A extensão possibilita ao aluno maior envolvimento acadêmico e social na própria universidade. Os preparativos e a realização das ações da extensão geram interações entre os alunos, viabilizam novos conhecimentos decorrentes da troca de ideias, 
promovem envolvimento com as comunidades e incentivam a desenvoltura acadêmica através da participação em congressos a partir da extensão.

\subsection{Ações realizadas na extensão}

Em decorrência da pandemia da COVID-19 em 2020 e 2021 foram realizadas reuniões semanais com os alunos através dos meios virtuais. As atividades realizadas promoveram, na comunidade e nos alunos, diálogos e articulações do pensamento em relação à experiência vivida, gerando alternativas de melhoria da condição humana e social. Não houve atividades presenciais.

Realizou-se a produção de materiais de natureza técnico-cultural versando sobre os objetivos da proposta, como: vídeos, materiais informativos e outros. Os alunos participam de um ambiente de desenvolvimento de muitas competências, como: trabalho em equipe, comunicação, diálogo, protagonismo, dentre outros.

As ações realizadas deram ganho de competências nos alunos e os colocou diante de outras realidades, ao mesmo tempo em que lhes possibilitou melhores condições de vida para a comunidade.

Houve a participação em um evento disponibilizado pela Universidade, chamado "Mostra de Inovação e Empreendedorismo", que visa estimular os alunos a participarem do universo de "startups", com dois projetos nos quais os alunos desenvolveram produtos de engenharia para serem aplicados nas comunidades. Ocorreu boa dedicação de tempo por parte dos alunos.

\subsection{Grupos de alunos voluntários da Extensão}

Com o aumento do número de alunos participantes, houve o favorecimento do trabalho em equipe multidisciplinar e a formação de vários grupos de trabalho, beneficiando a interação e o diálogo entre todos.

No $1^{\circ}$ semestre o "Grupo de Alunos Voluntários da Extensão" contou com 22 alunos dos cursos de Engenharia e no $2^{\circ}$ semestre com mais 13 alunos.

A Tabela 1 mostra os alunos participantes dos diferentes cursos de engenharia. No segundo semestre houve a desistência de um aluno, a incorporação dos demais se deu a partir do convite dos colegas e pelo interesse de alunos do $1^{\circ}$ ano de Engenharia de Computação, totalizando 35 alunos diferentes.

Tabela 1 - Número de alunos no Projeto de Extensão.

\begin{tabular}{|c|c|c|c|}
\hline Curso & Ano & $1^{\circ}$ semestre & $2^{\circ}$ semestre \\
\hline Engenharia Elétrica & $2^{0}$ & 6 & 8 \\
\hline Engenharia de Computação & 10 & - & 10 \\
\hline Engenharia de Computação & $2^{0}$ & 7 & 7 \\
\hline Engenharia de Computação & $3^{0}$ & 8 & 9 \\
\hline Engenharia de Produção & $3^{0}$ & 1 & - \\
\hline & Total & 22 & 34 \\
\hline
\end{tabular}

Fonte: Os Autores.

No Quadro 1 está a organização das tarefas que foi realizada inicialmente no 1은 semestre com três grupos ou frentes de trabalho e, no segundo semestre um 4ำ grupo, resultando em quatro frentes de trabalho. No Quadro 1 está indicado como "maioria" a preponderância dos alunos participantes no grupo de acordo com os cursos de engenharia.

Quadro 1 - Grupos de trabalho 


\begin{tabular}{|c|c|c|}
\hline 1 & $\begin{array}{l}\text { Ações de conscientização sobre uso } \\
\text { racional e eficiência energética. } \\
\text { Maioria: } 2^{\circ} \text { ano - Engenharia Elétrica }\end{array}$ & $\begin{array}{l}\text { Criação de materiais informativos. } \\
\text { Apoio aos artigos e resumos para Congressos e } \\
\text { elaboração de vídeos }\end{array}$ \\
\hline 2 & $\begin{array}{l}\text { Possibilitar a implantação de soluções } \\
\text { (projetos, produtos e serviços) às } \\
\text { comunidades. } \\
\text { Maioria: } 2^{\circ} \text { ano - Eng. de Comp. }\end{array}$ & $\begin{array}{l}\text { Implantação de soluções. } \\
\text { Materiais informativos. } \\
\text { Apoio aos artigos e resumos para Congressos e na } \\
\text { elaboração de vídeos. }\end{array}$ \\
\hline 3 & $\begin{array}{l}\text { Dar apoio às ações da Extensão. } \\
\text { Maioria: 3ํa - Eno. de Comp. }\end{array}$ & $\begin{array}{l}\text { Elaboração de vídeos, criação e administração de um } \\
\text { site, e criação de um canal no "YouTube". }\end{array}$ \\
\hline 4 & $\begin{array}{l}\text { Produtos voltados à eficiência } \\
\text { energética para as comunidades. } \\
\text { A partir do } 2^{\circ} \text { semestre de } 2020 \text {. } \\
\text { Maioria: } 1^{\circ} \text { ano - Eng. de Comp. }\end{array}$ & $\begin{array}{l}\text { Produto 1: Dispositivo Fotovoltaico. } \\
\text { Produto 2: Acionamento de um ponto de luz e } \\
\text { informações de presença. } \\
\text { Produto 3: Gerenciamento de pontos de iluminação. }\end{array}$ \\
\hline
\end{tabular}

Fonte: Os Autores.

\subsection{Reuniões de orientações}

Foram realizadas reuniões de orientação com periodicidade semanal para cada um dos grupos, cada reunião com duração de cerca de uma hora. Utilizou-se a plataforma "Office365", disponibilizada pela PUC-Campinas, na qual foram criadas no "Microsoft Teams" quatro salas, uma para cada grupo. Outros meios de interação também foram utilizados, como: WhatsApp e e-mail.

O Projeto de Extensão criou um e-mail para disponibilizar os materiais informativos e um repositório na nuvem para facilitar a interação dos trabalhos entre os alunos de cada grupo e entre todos.

Os alunos foram se adequando aos trabalhos com liberdade para atuarem em cada um dos grupos, fato que possibilitou maior sinergia. Cada aluno atuava onde se sentia à vontade ou tinha interesse. Para eles, o trabalho colaborativo possibilitou melhor interação e compreensão com a Extensão. Os resultados foram bons, como: produção acadêmica, com artigos publicados em anais de Congressos, elaboração de materiais informativos de apoio à comunidade, além de diversos vídeos.

\section{GRUPOS DE TRABALHO E SINERGIA}

A curricularização da extensão universitária impulsionará muitas frentes de trabalho e o número de participantes deverá aumentar significativamente. Experiências no gerenciamento e na coordenação são fundamentais para a obtenção de bons resultados das ações. Na extensão é muito importante a produção colaborativa e em equipe.

Seguem algumas considerações sobre o trabalho em equipe, tendo em conta que na engenharia o planejamento, a divisão de tarefas e a condução de equipes ou grupos de trabalho são importantes. No trabalho em equipe há o exercício e a prática de várias de competências, tais como: liderança, responsabilidade, planejamento para aperfeiçoar a execução, orientação de tarefas, acompanhamento das tarefas, treinamento, dentre outras.

Normalmente um grupo de trabalho apresenta participantes com diferentes personalidades, experiências e áreas. $O$ engenheiro ao se exercitar na engenheira deve saber trabalhar em grupos heterogêneos, com isso, sua carreira ganha desenvoltura ao dialogar com a diversidade de integrantes, tornando mais fácil lidar com os problemas e as soluções.

Para a resolução de problemas ou para os desafios, muitas vezes ocorre uma divisão de tarefas e de responsabilidades entre os membros do grupo. Essa divisão é realizada por um líder ou coordenador que acompanhará o projeto com planejamento, 
desde o início das ações, delegando tarefas e estabelecimento de sinergia entre os participantes do grupo, até o final do projeto ou do trabalho.

\subsection{Equipes multidisciplinares}

As equipes multidisciplinares contam com integrantes de diversas áreas, cada participante pode possuir domínio acerca de diferentes assuntos e, com isso, apresentar diferentes perspectivas acerca do objetivo da equipe. Em uma equipe multidisciplinar não há hierarquia, entretanto, é necessária a presença de um coordenador a fim de manter harmonia entre os membros e alcançar os objetivos propostos.

Além disso, essa formação de equipe conta com uma vasta troca de informações, sendo essencial uma comunicação fluida e constante entre todos para que haja sincronia e compromisso da equipe em todos os processos do projeto, a fim de alcançar um resultado com maior criatividade e enriquecimento de ideias e soluções. (SILVA, 2015)

\subsection{Planejamento e delegação}

O planejamento é uma parte essencial em um projeto ou trabalho, momento em que a equipe se reúne para a idealização e definição do projeto: definem-se as dataslimite, os objetivos, quem e de qual maneira serão desenvolvidas as abordagens. Para maior probabilidade de sucesso e produtividade sugere-se que a equipe que o planeja seja a mesma que o desenvolverá.

Definir metas e prazos é a base para o bom planejamento.

Antigamente o coordenador de um grupo de trabalho era visto como centralizador, onde era necessária a capacidade de coordenar e se comunicar claramente. Apenas se descentralizava pequenas partes do projeto delegando-as para os demais participantes.

As novas tendências de grupos de trabalho concentram esforços para a criação de um ambiente que incentiva a todos contribuírem para o objetivo, onde a capacidade do coordenador de lidar com as pessoas é sua maior responsabilidade social, também chamado de "empowerment". Esse conceito é definido por Slack (2002) como "dar ao pessoal a autoridade para fazer mudanças no trabalho em si, assim como na forma ele é desempenhado", ou seja, os líderes devem desistir de terem o projeto centralizado em si para dar a autoridade a todos os subordinados.

No processo de ocorrer a delegação, o coordenador deve estar aberto para aceitar ideias, transferir o poder de decisão da tarefa aos demais membros, tolerar erros, confiar e recompensar uma tarefa bem-sucedida (CARDOSO, 1998).

\subsection{Acompanhamento de tarefas}

Estabelecer o acompanhamento ou gestão de tarefas é um processo essencial para conseguir setorizar cada etapa do desenvolvimento e, através do acompanhamento, visualizar de maneira mais clara e específica a trajetória da tarefa.

O acompanhamento das tarefas ou dos trabalhos, garante que haja fluxo do que está sendo realizado de acordo com metas e prazos. $\mathrm{O}$ bom planejamento fundamentado na experiência própria ou de outros colabora diretamente para a definição das etapas a serem realizadas facilitando o acompanhamento das tarefas.

Para facilitar essas análises, pode-se utilizar diferentes ferramentas, como sistemas e aplicativos que viabilizam diferentes modelos de uso como métodos de gestão e organização.

\subsection{Sinergia do grupo}

A sinergia tem um papel fundamental dentro do desenvolvimento de tarefas. Colabora para a sinergia a maneira em que os participantes do grupo se organizam para 
a realização das atividades. É importante que haja a estimulação da equipe para que se tenha um bom entrosamento. Em vista disso, os passos importantes para gerar esse estímulo são: a criação de um plano estratégico, avaliação dos participantes e definição dos padrões da equipe.

O estilo de trabalho definido pelo grupo estabelece o ritmo e facilita para que todos os participantes fiquem em harmonia a fim de alcançar melhores resultados, ou seja, quanto mais sinergia presente entre os participantes, mais efetivo será o desempenho durante a realização da tarefa.

Como consequência da definição do estilo ocorre a geração de melhores resultados por conta da melhor comunicação e entrosamento entre o grupo. A sinergia acaba beneficiando o resultado do trabalho e os participantes por tornar a participação mais gratificante.

\section{PERCEPÇÃO DOS ALUNOS DIANTE DOS GRUPOS DE TRABALHO}

Foi elaborado um questionário que foi enviado por e-mail ou "WhatsApp" aos alunos participantes da Extensão em 2020. O questionário era constituído por perguntas relacionadas ao modo de trabalho dos grupos e como, na percepção dos alunos, o trabalho ou a condução das equipes poderia dar melhor resultado. Dos 35 alunos, 24 responderam ao questionário.

$\mathrm{Na}$ Figura 1 os alunos avaliaram a forma com que trabalharam nas tarefas do Projeto Extensão. A partir das respostas pode-se observar que 83,3\% preferiram trabalhar em grupo ou equipe, indicando que estão voltados para um trabalho colaborativo.

Figura 1 - Respostas da Pergunta:

"Como você preferiu trabalhar na Extensão?"

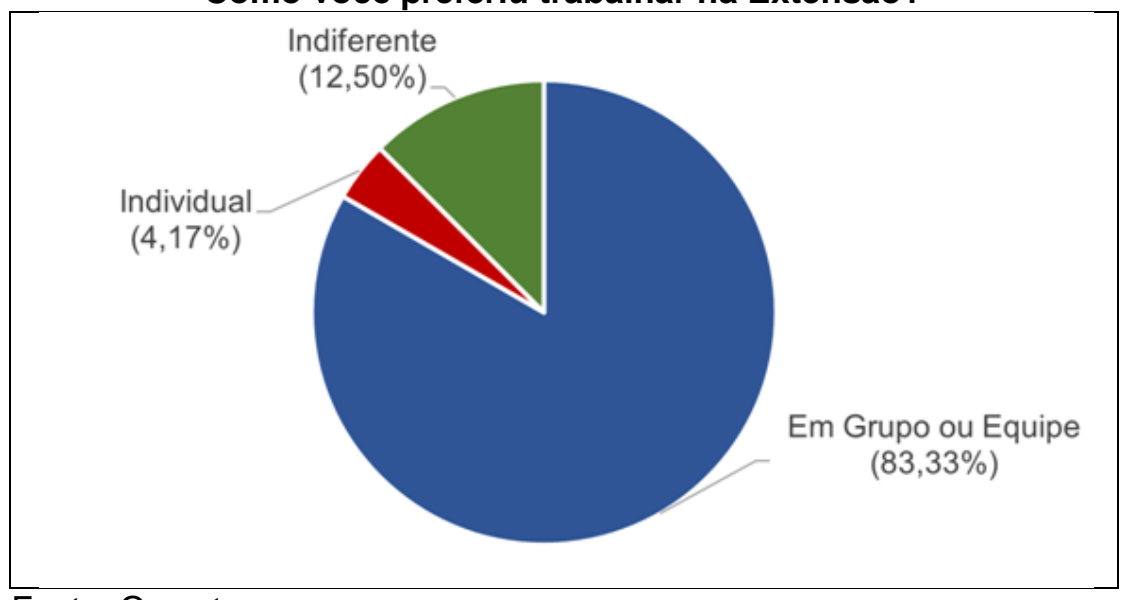

Fonte: Os autores.

Na pergunta sobre como obter melhor resultado de conjunto ou sinergia, na Figura 2 , pode-se observar que $58,4 \%$ dos alunos apontam que trabalhar num grupo menor poderá dar melhor resultado. Nas respostas, há destaque para os que são indiferentes $33,33 \%$ em relação a trabalhar num grupo menor ou maior.

Figura 2 - Respostas da Pergunta:

"No trabalho em equipe. Na sua opinião, o que poderia dar melhor resultado de conjunto (sinergia)?" 


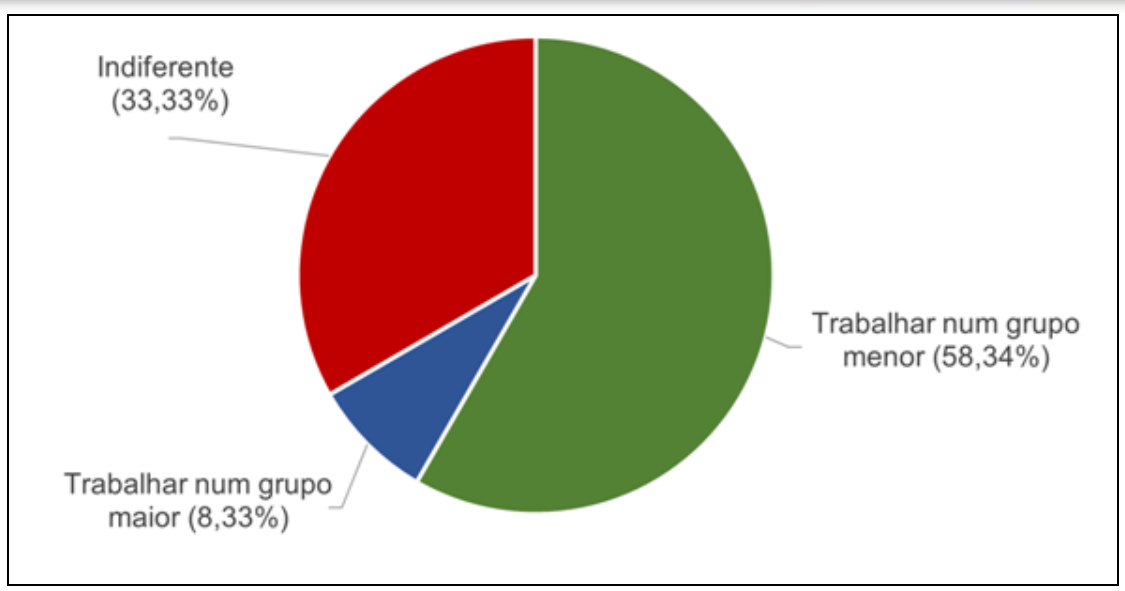

Fonte: Os autores.

$\mathrm{Na}$ Tabela 2 estão as respostas assinaladas pelos alunos de como poderia dar melhor resultado ou sinergia no trabalho em equipe, com a possibilidade de serem assinaladas mais de uma alternativa. Com base nas respostas obtidas, a maioria assinalou "consenso e troca de ideias para realizar as tarefas, e depois estabelecer um coordenador". Cerca de $44 \%$ das alternativas assinaladas, isto é, "a" (14\%), "c" (12\%) e "f" $(18 \%)$, reforçam que a presença do coordenador é importante, dando a entender que deve estar presente desde o primeiro momento. As metas e prazos, com 30\%, também favorecem a sinergia e estão presentes nas alternativas "c" (12\%) e "f" (18\%), e contribuindo para a objetividade, estabelecem "foco" de atuação para todos e marcam o compasso das tarefas e das ações.

Tabela 2 - Respostas da pergunta:

"Na sua opinião, como poderíamos obter melhor sinergia no trabalho em equipe?"

\begin{tabular}{|c|c|c|}
\hline \multicolumn{2}{|r|}{ Alternativas } & \multirow{2}{*}{$\begin{array}{c}\text { Quantidade } \\
\text { assinalada } \\
7 \\
\end{array}$} \\
\hline a) & O trabalho foi excelente com coordenador e equipe & \\
\hline b) & Ser o coordenador & 2 \\
\hline c) & Ter um coordenador exigente nos prazos e nas tarefas & 6 \\
\hline d) & $\begin{array}{l}\text { Consenso e troca de ideias para realizar as tarefas, e } \\
\text { depois estabelecer um coordenador }\end{array}$ & 13 \\
\hline e) & Revezamento de coordenador & 4 \\
\hline f) & $\begin{array}{c}\text { Coordenador externo (Ex.: professor), com prazos e metas para a } \\
\text { equipe }\end{array}$ & 9 \\
\hline g) & Equipe multidisciplinar (com colegas de outros cursos) & 5 \\
\hline h) & Delegar mais tarefas individuais para somar no trabalho & 4 \\
\hline & 10 & 50 \\
\hline
\end{tabular}

Fonte: Os autores.

$\mathrm{Na}$ Figura 4 estão as respostas assinaladas de uma forma mais visual com as respectivas porcentagens.

Figura 4 - Respostas da pergunta:

"Na sua opinião, como poderíamos obter melhor sinergia no trabalho em equipe?" 


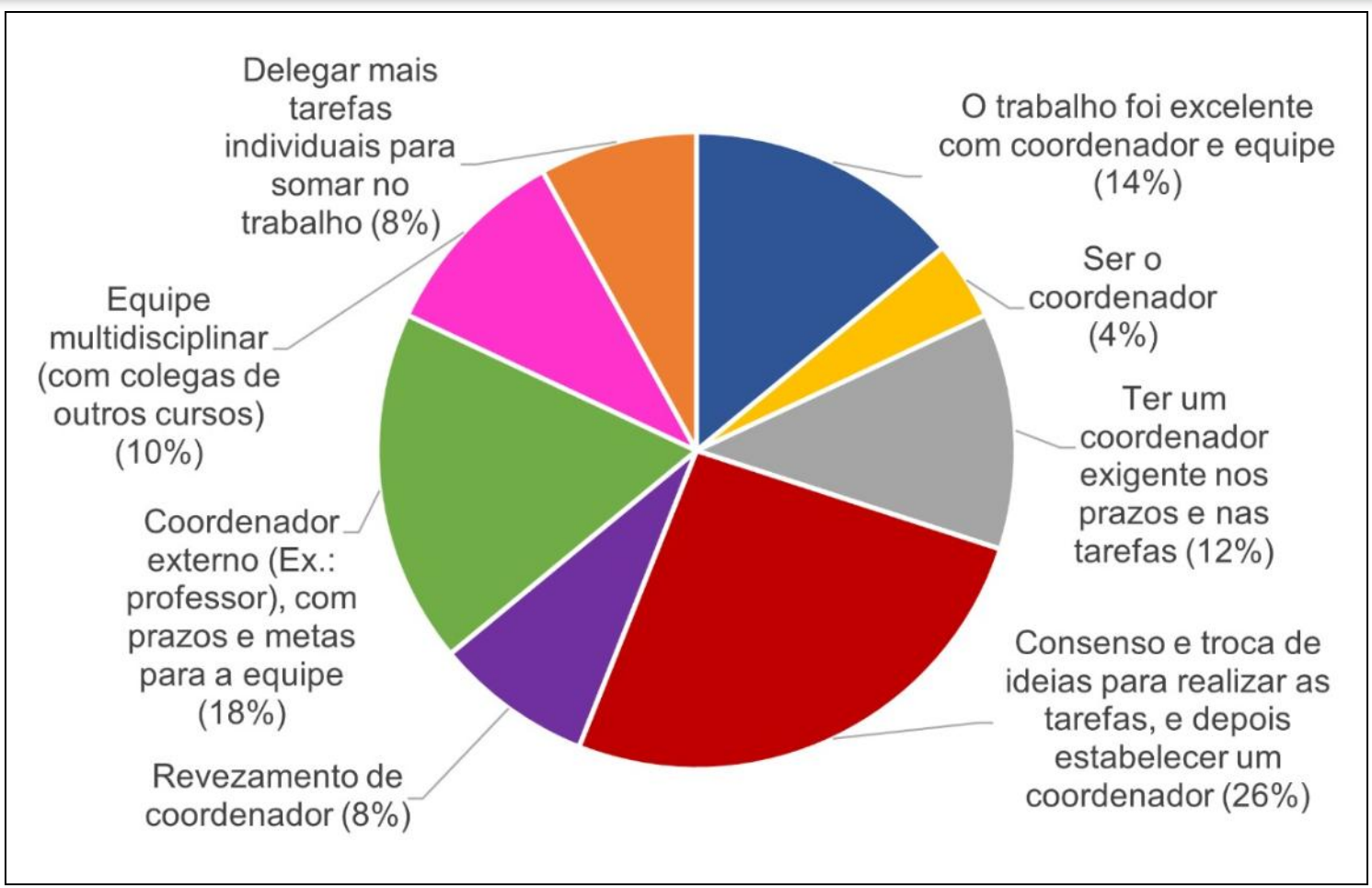

Fonte: Os autores.

Um questionamento foi feito por um aluno integrante do Projeto de Extensão, segue o seu relato:

"Eu achei que conseguimos alcançar os resultados esperados, assim como conseguimos entregar todas as tarefas propostas. Entretanto, senti um pouco de dificuldade na comunicação com os outros participantes da minha equipe, pois, muitas vezes apenas alguns participantes realizam sua parte e assim o coordenador da equipe ficava sobrecarregado. Além disso, senti que alguns colegas apenas ignoravam o que estava sendo pedido ou questionado pelo coordenador e só davam um feedback após várias cobranças."

Nesse relato ficam claros os fatos corriqueiros do trabalho em equipe como "dificuldade de comunicação com outros participantes", que o coordenador "ficava sobrecarregado" e que o retorno só ocorria após "várias cobranças". Os alunos estão no processo de formação profissional e no amadurecimento pessoal com possibilidade de desenvolver competências nas componentes curriculares do curso de engenharia, a extensão com suas atividades e com seus leques de possibilidades contribui eficazmente para essa formação de excelência.

Outros depoimentos ou relatos poderiam enriquecer o presente estudo além das perguntas com respostas de alternativas. Outros Projetos de Extensão poderiam dar outras percepções por parte dos alunos não só dos cursos de engenharia.

\section{CONCLUSÃO}

O aumento do número de alunos voluntários no projeto de extensão gerou possibilidades em diversas frentes nas quais os participantes foram escolhendo onde desejavam se dedicar. As respostas ao questionário realizado nos mostram suas preferências, dando condições de melhores atuações nos trabalhos de extensão com um número maior de pessoas. As perspectivas dos alunos podem auxiliar nas diretrizes dos trabalhos de extensão no que se refere à formação de grupos de trabalho. 
Com a pandemia da COVID-19 outras estratégias foram adotadas fazendo com que as ações virtuais ganhassem destaque no projeto de extensão, tais ações auxiliaram no desenvolvimento de capacidades dos alunos e contribuíram para desenvoltura tanto na formação acadêmica quanto fora da Universidade.

A formação de grupos de trabalho dá condições para prática da liderança de equipes e favorece a capacidade para trabalhar em equipes multidisciplinares, bem como, a de expor ideias para a execução do projeto e favorece o desenvolvimento do trabalho colaborativo.

No Projeto de Extensão as frentes associadas aos grupos de trabalho possibilitaram bons resultados. A gestão do grupo de trabalho por um aluno coordenador que auxiliava o professor e que acompanhava as atividades realizadas pelos colegas foi uma boa estratégia. Através do questionário percebe-se que a grande maioria dos alunos se sente mais à vontade em equipes do que com atuação individual e a preferência é para grupos menores. Para os alunos é importante a troca de ideias para se chegar a um consenso e, a presença de um coordenador também é importante, sendo ele externo ou integrante do grupo. Os alunos assinalaram que ter um coordenador com metas e prazos contribui para ter maior sinergia no trabalho.

\section{Agradecimentos}

À Pontifícia Universidade Católica de Campinas (PUC-Campinas) por todo apoio, que desde o primeiro momento viabilizou e contribuiu para tornar realidade o Trabalho de Extensão e aos alunos voluntários pelas valiosas contribuições.

\section{REFERÊNCIAS}

CARDOSO, Maria E. Trabalho em Equipe: Uma estratégia de gestão. Universidade Federal de Santa Catarina, Florianópolis, 1998. Disponível em:

https://repositorio.ufsc.br/bitstream/handle/123456789/104500/Trabalho\%20em\%20equipe \%20uma\%20estrat\%C3\%A9gia\%20de\%20gest\%C3\%A3o.pdf?sequence=1\&isAllowed=y. Acesso em: 22 abr. 2021

FERNANDES, Mônica Abranches. Trabalho Comunitário: Uma Metodologia para Ação Coletiva e educativa da Extensão Universitária em Comunidades. In: MENEZES, Ana Luisa Teixeira e SÍVERES, Luiz. Transcendendo Fronteiras a Contribuição da Extensão das Instituições Comunitárias de Ensino Superior (ICES). Santa Cruz do Sul: EDUNISC, 2011 p. [138-158].

FREIRE, Paulo. Pedagogia da Autonomia. 30ª ed. São Paulo: Paz na Terra, 2004.

PUC-CAMPINAS. Pontifícia Universidade Católica de Campinas. Missão da

Universidade. Disponível em: <https://www.puc-campinas.edu.br/institucional/reitoria/>. Acesso em: 20 mai. 2020.

REIS, Thiago. Sinergias: Descubra o que é e veja 3 exemplos reais de sinergias. Disponível em: https://www.suno.com.br/artigos/sinergias/. Acesso em 21 abril 2021.

SILVA, Cilano M. Equipe Multidisciplinares. RH Site, 2015. Disponível em: https://www.rhportal.com.br/artigos-rh/equipes-multidisciplinares/. Acesso em: 23 abr. 2021 
SLACK, N. et al. Administração da Produção. 2ª ed. São Paulo: Editora Atlas, 2002.

\title{
WORKING GROUPS ON THE EXTENSION PROJECT AND THE STUDENTS' PERCEPTION TO IMPROVE TASK COORDINATION
}

\begin{abstract}
The Extension Project on Energy Efficiency had a significant increase in the number of participating students in 2020, from 8 in 2019 to 35 . The way of working adopted was to divide the actions into working groups. With the university extension curriculum, the number of participants is expected to increase significantly. The purpose of this article is to present the perceptions of the students who participated in the work groups of the Extension Project. As a methodology, a questionnaire was developed with questions for students to expose their perceptions. In the results, the answers to the questionnaires are presented with the students' perception of the work done in a team and their suggestions to improve coordination. In conclusion, most students prefer to work in smaller teams. The formation of work groups provides conditions for the practice of team leadership and favors the ability to work in multidisciplinary teams, as well as, exposing the possibility of exposing ideas for the execution of the work, favoring the development of collaborative work. An integral part of the Extension's actions is the joint production of material of a technical-cultural nature, as a way of improving the autonomy of the target audience and facilitating a wider range of knowledge acquired for other audiences. The Extension Work is carried out in conjunction with the Dean of Extension and Community Affairs of the Pontifical Catholic University of Campinas, with the participation of partner communities.
\end{abstract}

Keywords: University Extension. Student Academic Formation. Energy Efficiency. Engineering Skills. 